\title{
Heat Flux Modulation in Domino Dynamo Model
}

\author{
M. Reshetnyak ${ }^{1}$, P. Hejda ${ }^{2}$ \\ ${ }^{1}$ Institute of the Physics of the Earth, Russian Academy of Sciences, Moscow, Russia \\ ${ }^{2}$ Institute of Geophysics, Czech Academy of Sciences, Prague, Czech Republic \\ Email: m.reshetnyak@gmail.com,ph@ig.cas.cz
}

Received 2013

\begin{abstract}
Using the domino dynamo model, we show how specific axisymmetric and equatorial symmetric forms of the heat flux variations at the core-mantle boundary change the frequency of the geomagnetic field reversals. In fact, we are able to demonstrate the effect known from the modern 3D planetary dynamo models using an ensemble of interacting spins, which obey equations of the Langevin type with a random force. We also consider applications to the giant planets and offer explanations of some specific episodes of the geomagnetic field in the past.
\end{abstract}

Keywords: Liquid Core; Geomagnetic Field Reversals; Anisotropic Heat Flux; Thermal Traps

\section{Introduction}

Generation of the planetary magnetic fields is a subject of the dynamo theory, which describes successive transformations of thermal and gravitational energy, concerning compositional convection, to energy of kinetic motions of the conductive liquid and then to the energy of the magnetic field [1]. Modern dynamo models include partial differential equations of thermal and compositional convection as well as the induction equation for the magnetic field, which for some reasons should be three dimensional [2].

Although, due to the finite conductivity of the Earth's mantle, observations of the geomagnetic field at the Earth's surface are bounded by the first thirteen harmonics in the spherical function decomposition, one needs small- scale resolution down to $\approx 10^{-8} \mathrm{~L}$, to provide the necessary force balance in the core. Here $L=r_{o}-r_{i}=2270 \mathrm{~km}$ is the thickness of the fluid outer core and $r_{o}=3485 \mathrm{~km}, \mathrm{r}_{\mathrm{i}}$ $=1215 \mathrm{~km}$ are its boundaries. This difficulty is caused by the huge hydrodynamic Reynolds number $\mathrm{Re} \approx 10^{9}$ as well as by the strong anisotropy of convection [3] due to the geostropic state in the core [4]. Convection in the core is cyclonic. The cyclones and anticyclones are aligned with the axis of rotation, and their scale is much smaller than their length. As a result one needs very efficient computer resources to produce regimes in the desired asymptotic limit required for geodynamo simulations with grids $128^{3}$ and more, which is a challenge even for modern supercomputers.

In spite of these technical problems, 3D dynamo models successfully mimic various features of the modern and ancient magnetic field including the reversals [5]. One of the important results of the dynamo theory is that the frequency of the reversals depends on the intensity of the heat flux at the outer boundary of the liquid core [6] and, moreover, on the heterogeneity of the boundary flux $[7,8]$. In particular, it has been shown that the increase of the heat flux along the axis of rotation from the equator plane to the poles (see Figure 1(d) in [7]) leads to increase of the axial symmetry of the whole system and stops reversals. In a sense, the thermal trap of reversals occurs. On the contrary, the decrease of the thermal flux at high latitudes leads to the chaotic behavior of the magnetic dipole accompanied by frequent reversals, which is closely connected with the upsetting of the geostrophic balance and predominance of the radial (in an incompressible medium in which the parameters depend only on the radius) Archimedean forces. It looks attractive to obtain this result using toy dynamo models (such as the Rikitake and Lorenz models), which can provide extensive statistics and obviousness of the results, and simulate practically instantly, using just home PC, the number of reversals and excursions of the same order as known from paleomagnetism. Such simplified models has been used for the simulation of the Earth and planetary [9-12], as well as solar [13] magnetic fields. The random force, which imitates the small-scale unresolved fluctuations, was also included in some of these models [14]. We have selected the domino dynamo model $[15,16]$, which is an extension of the IsingHeisenberg XY-models of interacting magnetic spins. For more details of the history of the problem and classification refer to [17]. 

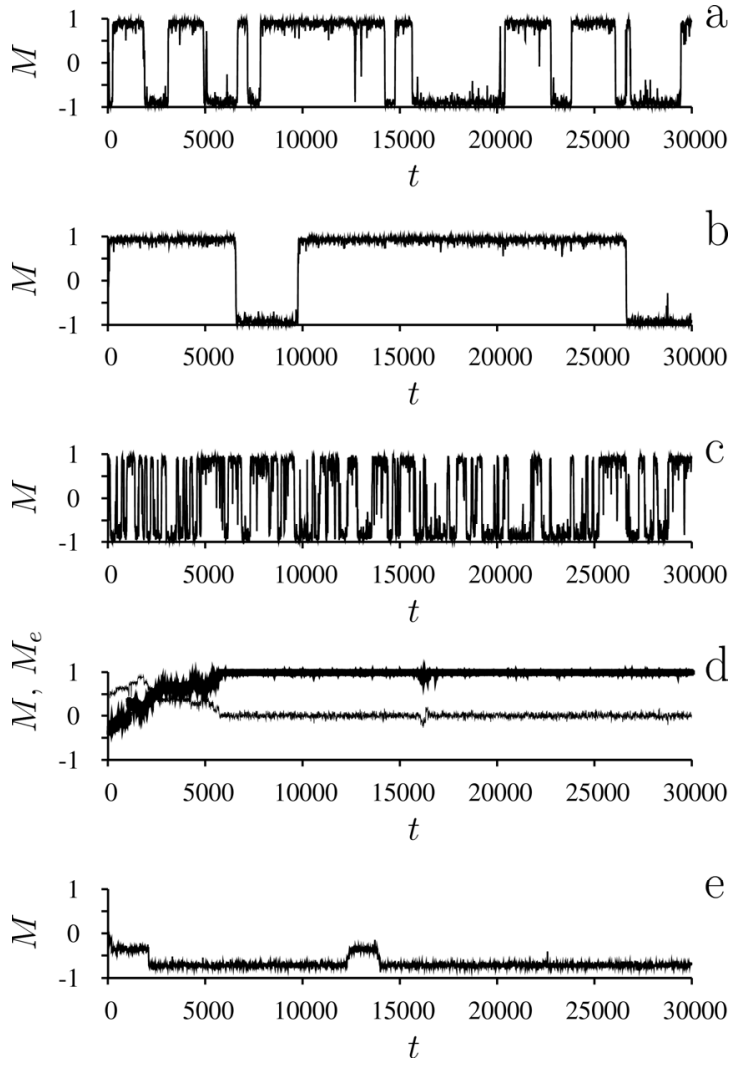

Figure 1. Evolution in time of the magnetic moment $M$ : (a) for the purely magnetic system $\left(C_{\psi}=0\right)$, (b) $C_{\psi}=0.5$, (c) $C_{\psi}$ $=-0.5$ for $\psi=-\cos ^{2} \theta$, (d) $C_{\psi}=10$, (e) $C_{\psi}=-9$ for $\psi=-\cos ^{2} 2 \theta$. At (d) the thick line corresponds to $M$ and the thin line to $M_{e}$.

\section{Domino Model}

The main idea of the domino model is to consider a system of $N$ interacting spins $\mathbf{S}_{\mathrm{i}}, \mathrm{I}=1 \ldots N$, embedded in media rotating with angular velocity $\mathbf{\Omega}=(0,1)$ in the Cartesian system of coordinates $(\mathrm{x}, \mathrm{y})$. The spins are evenly distributed along an equatorial ring, are of unit length and can vary angle $\theta$ from the axis of rotation in the range of $[0,2 \pi]$ on time $t$, so that $\mathbf{S}_{\mathrm{i}}=\left(\sin \theta_{\mathrm{i}}, \cos \theta_{\mathrm{i}}\right)$. Each spin $\mathbf{S}_{\mathrm{i}}$ is forced by a random force, effective friction, as well as by the closest neighboring spins $\mathbf{S}_{\mathrm{i}-1}$ and $\mathbf{S}_{\mathrm{i}+1}$.

Following [15], we introduce kinetic $K$ and potential $U$ energies of the system:

$$
\begin{aligned}
& K(t)=\frac{1}{2} \sum_{i=1}^{N} \dot{\theta}_{i}^{2}, \\
& U(t)=\gamma \sum_{i=1}^{N}\left(\Omega \cdot \mathrm{S}_{i}\right)^{2}+\lambda \sum_{i=1}^{N}\left(\mathrm{~S}_{i} \cdot \mathrm{S}_{i+1}\right)
\end{aligned}
$$

The Lagrangian of the system then takes the form $L=$ $K-U$. Transition to the Lagrange equations with friction proportional to velocity $\dot{\theta}$, and the random force $\chi$,

$$
\frac{\partial}{\partial t} \frac{\partial}{\partial \dot{\theta}}=\frac{\partial L}{\partial \theta}-\kappa \dot{\theta}+\frac{\varepsilon \chi}{\sqrt{\tau}},
$$

leads to the system of the Langevin-type equations:

$$
\begin{gathered}
\ddot{\theta}-2 \gamma \cos \theta_{i} \sin \theta_{i}+\lambda\left[\cos \theta_{i}\left(\sin \theta_{i-1}+\sin \theta_{i+1}\right)\right. \\
\left.-\sin \theta_{i}\left(\cos \theta_{i-1}+\cos \theta_{i+1}\right)\right]+\kappa \dot{\theta}_{i}+\frac{\varepsilon \chi}{\sqrt{\tau}}=0 \\
\theta_{0}=\theta_{N}, \theta_{N+1}=\theta_{1}, i=1 \ldots N,
\end{gathered}
$$

where $\gamma, \lambda, \kappa, \varepsilon, \tau$ are constants. The measure of synchronization of the spins along the axis of rotation

$$
M(t)=\frac{1}{N} \sum_{i=1}^{N} \cos \left(\theta_{i}(t)\right)
$$

will be considered to be the total axial magnetic moment. Integrating (3) in time for small $N$ one can arrive at quite diverse dynamics of $M$, very close (for some special choice of parameters) to paleomagnetic observations [17], including the periods of the random and frequent reversals.

It was shown in [15] that large fluctuation of a single spin can be successively transferred to the neighboring spins, which fluctuate until all spins reverse their polarity. This domino effect was an inspiration for the name "domino model". By definition this model can generate the magnetic field which can not die at all, because of the magnitudes of the individual spins are fixed. However, it is able to mimic synchronization of the individual spins producing the mean net flux of the magnetic field, the analog of the observed planetary mean field. Note that the idea of the cyclone synchronization is not new, see development of toy $\alpha \omega$-models $[11,12]$. However domino models are free from any mean-field assumptions, such as separation in scales, which is not so easy to combine with ideas of turbulent cascades, isotropy of the fields in presences of the rapid rotation and so on, that is why on our opinion domino model deserves special discussion.

In all simulations we have used, similarly to [15], values of parameters $\gamma=-1, \lambda=-2, \kappa=0.1, \varepsilon=0.65, \tau=10^{-2}$, $N=8$, with random normal $\chi_{i}$, with zero mean values and unit dispersions, so that $\chi_{i}$ was updated at every time step equal to $\tau$. The particular choice of $N$ merits special attention. As follows from analysis in [15], for the other fixed parameters, and $N=8$, the typical times of the system are more realistic. Evolution of $M$ depends slightly on the form of the random forcing $\chi$ and the remaining parameters can be easily selected in such a way as to provide similarity with observations. The typical behavior of the axial dipole is presented in Figure 1(a), where we observe 17 reversals at irregular time intervals. The meaning of the external force defined by parameter $C_{\psi}$, mentioned in Figure 1, will be specified latter and is assumed to be zero before discussion. Taking $3 \times 10^{5} \mathrm{y}$ for the mean time interval between the reversals we come to the estimate of the whole time interval as $510^{6} \mathrm{y}$ and time unity $\tau_{u}=160 \mathrm{y}$. Estimate of $\dot{\theta}=0.05$ leads to the typical time variations of the magnetic pole 


$$
\tau_{1}=\frac{\pi}{8 \dot{\theta}} \tau_{u} \sim 1200 y,
$$

where we assumed that between the reversals the magnetic pole is located in the cone $\theta<\frac{\pi}{8}$. This estimate seems to be reasonable and coincides with the estimate of the west drift velocity, archeomagnetic time scale and typical time of the magnetic pole wondering around the geographical pole. Ratio $\frac{\gamma \sqrt{\tau}}{\varepsilon} \sim 10$ corresponds to the dominance of the Coriolis force over the disturbances caused with the external forcing, e.g. temperature fluctuations. Neglecting spatial structure of the forces one gets magnetostrophic balance $\frac{2 \gamma}{\lambda}=1$ assuming that the $\lambda$-term in (3) has the magnetic origin.

Process of reversals is accompanied by the short drops of $M$ to nearly zero and followed by rapid recovers, which in geomagnetism are referred to as excursions of the magnetic field. One can find a thorough analysis of this system in [15]; here we only emphasize one important in geomagnetism point. Up to now observations do not clearly indicate, if the geomagnetic dipole just rotates during the reversal without decreasing the amplitude, or if it decreases and then recovers with the opposite sign. To check these scenarios we present the evolution of the individual spins during the reversal, see Figure 2. There are quite large deviations of the individual spins from the mean value $M$ at the moment of reversal $M=0$. This means that the decrease of $M$ is caused by the desynchronization of the spins rather than by the coherent rotation of the spins. Note that the typical time of the reversal is much larger than the time step. The points on the lines correspond to every $10^{\text {th }}$ time step in the simulations.

The other point is that the minimal time $\sim N \tau / 2$ of propagation of the disturbance from spin $\mathbf{S}_{\mathrm{i}}$ through half of the circle is also smaller than the typical reversal time. This scenario is also supported by the 3D simulations, where the spots of the magnetic field with opposite polarities co-exist at the core-mantle boundary during the reversals.

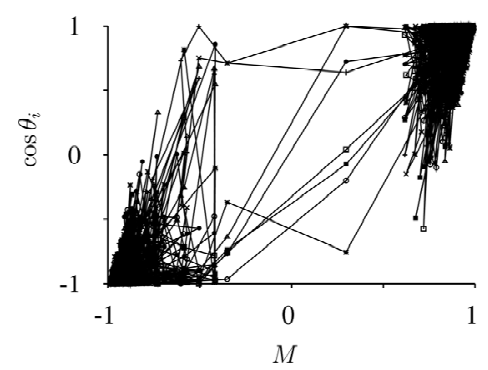

Figure 2. Plots of $\cos \theta_{\mathrm{i}}$ with $i=1 \ldots N$, versus $M$ for reversal in Fig.1a during the time interval $t=10^{3}-310^{3}$.

\section{Heterogeneous Heat-flux at the Core-mantle Boundary}

We now extend the concept of the spin from the purely magnetic system to the whole cyclone system, including its hydrodynamics, and we introduce the local correction $\Psi_{\mathrm{i}}$ to the potential energy $U_{\mathrm{i}}$ of $i^{\text {th }}$ spin, which takes into account the heterogeneity of the thermal flux. It will allow to compare our simulations with the above mentioned results reported in [6-8]. The new effective Force $F_{i}=-\frac{\partial \Psi_{i}}{\partial \theta_{i}}$, whose influence on the behavior of $M(t)$ will be considered in the rest of the paper, then appears in the right-hand side of (3). Let $\Psi(\mathrm{t}, \theta)=\mathrm{C}_{\psi} \psi(\mathrm{t}$, $\theta$ ), where $C_{\psi}$ is a constant, and the spatial distribution of the potential is given by $\psi=-\cos ^{2} \theta$. Accordingly to the recent estimates of the heat-flux variations at the core-mantle boundary, which can be about $20 \%$ [8], we conclude that $C_{\psi}=0.2 \frac{\varepsilon}{\sqrt{\tau}} \sim 1$. This estimate is a little bit larger of the variations used in [7]. Due to simplicity of our model we are able to consider only axi-symmetrical heat flux variations, which nevertheless found recent applications in the geodynamo modeling during Pangea formation [18]. Then, $\mathrm{C}_{\psi}>0$ corresponds to the stable state in the polar regions, $\theta=0, \pi$, and the appearing force $F=-\sin 2 \theta$, acting on the cyclones, is directed towards the poles. This regime corresponds to the increase of the thermal flux near the poles that causes the stretching of the cyclone along the axis of rotation. So as $F$ changes sign at the pole $\theta=\pi$, and the force is directed to the pole (or outward) for $\theta \rightarrow \pi-0$ and $\theta \rightarrow \pi+0$, we can consider it as the spherical coordinate when it is needed. In Figure 1 we demonstrate the effective influence of $F$ on $M$.

The increase of the thermal flux along the axis of rotation leads to the partial suppression of the reversals of the field, Figure 1(b). Note that, for the chosen potential barrier $\psi$, the dependence of $F$ is equal to the $\gamma$-term in (3): the increase of the thermal flux at the poles leads to the effective increase of rotation and amplification of geostrophy, caused by the rapid daily rotation of the planet. Our results are in agreement with the $3 \mathrm{D}$ simulations, see Figure 1(d) in [7]. The further increase of $\mathrm{C}_{\psi}$ $\left(C_{\psi}=2\right)$ leads to the total stop of the reversals. It is more interesting that, using even larger $\mathrm{C}_{\psi}>10$, one arrives at regimes with a nearly constant in time $|M|<1$ defined by the initial distribution of $\mathbf{S}_{\mathrm{i}}$. In other words, the super flux at the poles can fix the spins which are still not coherent. There is some evidence $[19,20]$ that the geomagnetic dipole in the past migrated from the usual position near the geographic poles to some stable state in the middle latitudes. Within the framework of our model, we can explain this phenomenon by the thermal super flux at the 
poles. Later we will discuss some other scenarios which yield similar results.

For negative $\mathrm{C}_{\psi}$, when the geostrophy breaks due to the relative intensification of convection in the equatorial plane, we get the opposite result, see Figure 1(c): the regime of the frequent reversals observed in Figure 1(c) in [7]. In this case force $F$ is directed from the poles and the equilibrium point at the poles becomes unstable. The new minimum of the potential energy at the equator leads to the appearance of a new attractor, so that for $\mathrm{C}_{\psi}=-5$ the axial dipole fluctuates with zero mean value and maximal amplitude $M \sim 0.4$. This state corresponds to the equatorial dipole.

$$
M_{e}(t)=\frac{1}{N} \sum_{i=1}^{N} \sin \left(\theta_{i}(t)\right)
$$

so that $\left|M_{\mathrm{e}}\right| \sim 1$ and does not undergo reversal. Similar behavior of the magnetic dipole is observed on Neptune and Uranus; for more details see, e.g. [21].

Now we consider $\psi=-\cos ^{2} 2 \theta$ for which the corresponding force $F=-2 \sin 4 \theta$ changes sign in each hemisphere, see the example with the second-order zonal spherical harmonic $-P_{4}^{0}$, where $P_{l}^{m}$ is associated Legendre polynomials, in Figure 1(e) in [7].

For $\mathrm{C}_{\psi}>0$, the potential barrier is in the middle latitudes, which prohibits the reversals as observed in Figure 1(e) in [7]. In one of our runs only one reversal was observed for $\mathrm{C}_{\psi}=2$. The other important point is the existence of the stable point at the equator, where $M=0$. We could then assume a regime with two attractors: near the poles and at the equator. This regime is really observed in Figure 1(d). The inverse transition was not observed. Inspection of the state with small $M$ at the beginning of the run leads to a quite large estimate of the equatorial dipole amplitude $M_{\mathrm{e}}$, see Figure 1(d). Thus, in principle, this regime can also be related to the giant planets' dynamo as well.

The last example corresponds to the case $\mathrm{C}_{\psi}<0$, when in addition to the attractors at the poles (related to rotation $\Omega$ ), two new attractors appear at the middle latitudes. The variation of $C_{\psi}$ leads to regimes $C_{\psi}=-1$ with frequent reversals, observed in Figure 1(f) in [7]. Moreover, we can get regimes, see Figrue 1(e), when the magnetic pole stays at the high latitudes, however, $|M| \neq 1$ (the partial synchronization of the spins). There are some jumps to the unstable state $M=0$ with the dipole at the equator. Note that the decrease of the spatial scale of $\psi$ leads to the increase of its amplitude $C_{\psi}$.

\section{Conclusions}

To conclude, let us stress that the toy models do not pretend to compete with the known 3D dynamo models. However, toy models are being developed for better understanding of physics of the processes. The main point is that model of the spins does have a background based on our present knowledge of the flow structure in the core. The system of the cyclones in the core, which act like the individual magnets, produces the net magnetic flux observed outside the volume of generation. These individual magnets can interact with each other, feel the direction of angular rotation and are disturbed by the thermal-compositional convection forces. The domino model is based on the function decomposition related to the geostrophic state. It is the geostrophy, which is responsible for the cyclone formation along the axis of rotation.

\section{REFERENCES}

[1] G. Rudiger and R. Hollerbach, "The Magnetic Universe: Geophysical and Astrophysical Dynamo Theory," Wiley-VCHr, Weinheim, 2004.

[2] C. A. Jones, "Planetary Magnetic Fields and Fluid Dynamos," Annual Review of Fluid Mechanics, Vol. 43, 2011, pp. 583-614. doi:10.1146/annurev-fluid-122109-160727

[3] P. Hejda and M. Reshetnyak, "Effects of Anisotropy in the Geostrophic Turbulence," Physics of the Earth and Planetary Interiors, Vol. 177, 2009, pp.152-160. doi:10.1016/j.pepi.2009.08.006

[4] J. Pedlosky, "Geophysical Fluid Dynamics," SpringerVerlag, New York, 1987.doi:10.1007/978-1-4612-4650-3

[5] U. R. Christensen, "Zonal Flow Driven by Strongly Supercritical Convection in Rotating Spherical Shells," Journal of Fluid Mechanics, Vol. 470, 2002, pp. 115-133. doi: $10.1017 / \mathrm{S} 0022112002002008$

[6] P. Driscoll and P. Olson, "Superchron Cycles Driven by Variable Core Heat Flow," Geophysical Research Letters, Vol. 38, No. 9, 2011, p. L09304. doi:10.1029/2011GL046808

[7] G. A. Glatzmaier, R. S. Coe, L. Hongre and P. H. Roberts, "The Role of the Earth's Mantle in Controlling the Frequency of Geomagnetic Reversal," Nature, Vol. 401, 1999, pp. 885-890. doi:10.1038/44776

[8] P. L. Olson, R. S. Coe, P. E. Driscoll, G. A. Glatzmaier and P. H. Roberts, "Geodynamo Reversal Frequency and Heterogeneous Core-Mantle Boundary Heat Flow," Physics of the Earth and Planetary Interiors, Vol. 180, No. 1-2, 2010, pp.66-79. doi:10.1016/j.pepi.2010.02.010

[9] I. Melbourne, M. R. E. Proctor and A. M. Rucklidge, “A Heteroclinic Model of Geodynamo Reversals and Excursions," Dynamo and Dynamics, a Mathematical Challenge, Kluwer, Dordrecht, Vol. 26, 2001, pp. 363-370.doi:10.1007/978-94-010-0788-7 43

[10] D. A. Ryan and G. R. Sarson, "Are Geomagnetic Reversals Controlled by Turbulence within the Earth's core?" Geophysical Research Letters, Vol. 34, 2007, p. L02307. doi:10.1029/2006GL028291

[11] C. Narteau, J. L. Le Mouel, M. Shnirman, E. Blanter and C. Allegre, "Reversal Sequence in a Multiple Scale 
Dynamo Mechanism," Physics of the Earth and Planetary Interiors, Vol. 120, No. 4, 2000, pp. 271-287. doi:10.1016/S0031-9201(00)00145-X

[12] C. Narteau and J. L. Le Mouel, "Transient Evolution Regimes in a Multiscale Dynamo Model: Timescales of the Reversal Mechanism," Journal of Geophysical Research: Solid Earth, Vol. 110, 2005. p.B01104. doi:10.1029/2004JB002983

[13] D. Volobuev, "Toy Dynamo to Describe the Long-Term Solar Activity Cycles," Solar Physics, Vol. 238, No. 2, 2006, pp. 421-430. doi:10.1007/s11207-006-0154-x

[14] P. Hoyng, "Helicity Fluctuations in Mean Field Theory: An Explanation for the Variability of the Solar Cycle?" Astronomy \& Astrophysics, Vol. 272, 1993, pp. 321-339.

[15] N. Mori, D. Schmitt, J. Wicht, A. Ferriz-Mas, H. Mouri, A. Nakamichi and M. Morikawa, "Domino Model for Geomagnetic Field Reversals," Physical Review E, Vol. 87, 2013, p. 012108. doi:10.1103/PhysRevE.87.012108

[16] A. Nakamichi, H. Mouri, D. Schmitt, A. Ferriz-Mas, J. Wicht and M. Morikawa, "Coupled Spin Models for Magnetic Variation of Planets and Stars," Monthly Notices of the Royal Astronomical Society, Vol. 423, 2012, pp. 2977-2990. doi:10.1111/j.1365-2966.2012.20862.x

[17] H. E. Stanley, "Introduction to Phase Transitions and Critical Phenomena," Oxford University Press, Oxford,
1987.

[18] J. S. Gee and D. V. Kent, "Source of Oceanic Magnetic Anomalies and the Geomagnetic Polarity Reversals," In Treatise on Geophysics, Geomagnetism (ed. M. Kono), Elsevier, Amsterdam, Vol. 5, 2009, pp. 455-508.

[19] N. Zhang and S. Zhong, "Heat Fluxes at the Earth's Surface and Core-Mantle Boundary Since Pangea Formation and Their Implications fo the geomagnetic superchrons," Earth and Planetary Science Letters, Vol. 306, No. 3-4, 2011, pp. 205-216. doi:10.1016/j.eps1.2011.04.001

[20] A. Abrajevitch and Rob Van der Voo, "Incompatible Ediacaran paleomagnetic Directions Suggest an Equatorial Geomagnetic Dipole Hypothesis," Earth Planetary Science Letters, Vol. 293, 2010, pp. 164-170. doi:10.1016/j.eps1.2010.02.038

[21] A. V. Shatsillo, A. N. Didenko and V. E. Pavlov, "Two Competing Paleomagnetic Directions in the Late Vendian: New Data for The SW Region of the Siberian Platform," Russian Journal of Earth Sciences, Vol. 7, No. 4, 2005, p. ES4002. doi:10.2205/2004ES000169

[22] I. Cupal, P. Hejda and M. Reshetnyak, "Dynamo Model with Thermal Convection and with the Free-Rotating Inner Core," Planet. Space Science, Vol. 50, 2002, pp. 1117-1122. doi:10.1016/S0032-0633(02)00051-X 